\title{
Funciones cognitivas en la descendencia de pacientes con trastorno bipolar I
}

\author{
Humberto Carlos Bertieri Arcila a , Adonilso Julio De La Rosa b,d, Rocío Lemos Buitrago ${ }^{\mathrm{c}, \mathrm{d}}$
}

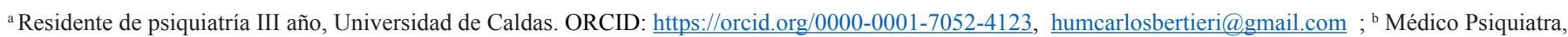
especialista en Psiquiatría infantil. Universidad de Caldas, Universidad de Manizales. ORCID: https://orcid.org/0000-0002-2272-8401, adonilso.julio@ucaldas. $\underline{\text { edu.co ; }}$; Psicóloga, Especialista en Neuropsicología clínica; d Docente Facultad de Ciencias para la Salud, Universidad de Caldas. ORCID: https://orcid.org/0000-0001-5259-2472, rocio.lemos@ucaldas.edu.co

DOI: https://doi.org/10.22517/25395203.23161

\section{Resumen}

Objetivo: Describir las características neuropsicológicas de niños y adolescentes descendientes de padres con antecedentes de TB I, respecto a la aplicación de una prueba estandarizada de inteligencia.

Materiales y métodos: Estudio observacional descriptivo de corte transversal, en donde los autores investigaron el Coeficiente Intelectual Total; Índice de compresión verbal; Índice de Razonamiento Perceptual; Índice de Memoria de trabajo; Índice de Velocidad de Procesamiento. Se evaluaron $(n=30)$ descendientes al aplicar varias subpruebas de la Escala de inteligencia de Weschler para niños, (WISC -IV).

Resultados: El Coeficiente Intelectual Total de los participantes con un $63,3 \%$ está por debajo del promedio de la curva de distribución normal, según la estandarización del test de inteligencia aplicado. Clasificando la tendencia de la muestra como un promedio normal bajo, en cuanto a la muestra evaluada. Los índices de memoria de trabajo y compresión verbal fueron las funciones cognitivas con puntuaciones más bajas en la muestra.

Discusión: Nuestros hallazgos sugieren un riesgo en hijos de pacientes con TBI, que puede contribuir a un incremento cuantitativo de las alteraciones cognitivas, especialmente el índice de memoria de trabajo y compresión verbal. Estos hallazgos pueden sugerir un posible endofenotipo del TB I y su descendencia, en relación a la presentación temprana de alteraciones cognitivas en este grupo de riesgo. Dichos hijos podrían ser un punto de partida para realizar estudios que determinen predicción y realizar así un abordaje temprano.

Palabras Clave: Trastorno Bipolar, Descendencia, Neuropsicología, Endofenotipo.
Psychopathological and neuropsychological profile in the offspring of patients with bipolar disorder type I

\begin{abstract}
Objective: To describe the neuropsychological characteristics of offspring children and adolescents of patients with bipolar disorder type 1 diagnosis through the application of a standardized intelligence test.
\end{abstract}

Materials and methods: In this descriptive crosssectional observational study, the authors investigated aspects such as Intelligence Quotient, Verbal Comprehension Index, Perceptual Reasoning Index, Working Memory Index, and Processing Speed Index. Here, 30 descendants were evaluated by applying several subtests of the Wechsler Intelligence Scale for Children (WISC-IV).

Results: The Total Intelligence Quotient of $63.3 \%$ of the participants is below the average of the Normal Distribution curve according to the standardization of the intelligence test applied which classifies the tendency of the sample as a low normal average. The working memory and verbal comprehension indexes were the cognitive functions with the lowest scores in the sample.

Discussion: Our findings suggest a risk in children of patients with Bipolar Disorder type I, which may contribute to a quantitative increase of cognitive disorders, especially in terms of working memory and verbal comprehension indexes. These findings may indicate a possible endophenotype of Bipolar Disorder type I patients and their offspring in regards to the early manifestation of cognitive disorders in this risk group. These children could be a target for studies that determine prediction and thus perform an early treatment.

Keywords: Bipolar Disorder, Offspring, Neuropsychology, Endophenotype. 


\section{Introducción}

El Trastorno Bipolar (TB) es un trastorno mental grave que afecta entre el 0,6 y el $2,4 \%$ de la población general (1) y el 1,2\% de los niños y adolescentes (2). La heredabilidad del trastorno se ha estimado entre 56 y $93 \%$ $(3,4)$, y el componente genético se ha considerado uno de los factores de riesgo más importantes para desarrollar el trastorno $(3,5)$. El riesgo de TB en la descendencia de los pacientes es de aproximadamente 4-5\%, aumentando a $14-35.8 \%$ si ambos padres son diagnosticados con TB $(6,7)$. Otros estudios describen estimaciones del riego más altas para el niño entre el 15-30\%; si ambos padres padecen TB el riesgo aumenta a $50-75 \%(8)$. Por lo tanto, la evaluación de la descendencia de niños y adolescentes de pacientes diagnosticados con TB brinda la oportunidad de investigar las primeras anormalidades $y$ factores de vulnerabilidad para la enfermedad en una muestra con mayor riesgo de trastorno mental y alteraciones cognitivas $(9,10,11)$.

Los hallazgos más consistentes, informados en muestras de adultos, han sido dificultades en la memoria verbal, la atención y las funciones ejecutivas $(12,13)$. Además, estas dificultades se han identificado durante el curso del trastorno (fase eutímica, aguda y crónica) $(14,15)$. En niños y adolescentes diagnosticados con $\mathrm{TB}$, la literatura sugiere un patrón similar de deterioro cognitivo al observado en poblaciones adultas. Los déficits en la memoria verbal, la atención y las funciones ejecutivas son las anormalidades neuropsicológicas más comúnmente reportadas $(16,17)$, siendo los endofenotipos muy importantes en el entendimiento de la importante carga genética.

Hallazgos a nivel neuropsicológico podrían considerarse como un endofenotipo de esta población o una caracterización neurocognitiva del trastorno, que permite una distinción más clara con otras entidades psiquiátricas o neurológicas con presentaciones fenomenológicas parecidas que implican dificultad diagnóstica $(18,19)$. La descripción de endofenotipo inicialmente formulado por Lewis y Gottesman en los años sesenta refería a un indicador de vulnerabilidad, no necesariamente expresado como marcador clínico de estado, sin embargo, este marcador subclínico era constitutivo en el fenotipo como un componente que cosegregaba con el background genético de la enfermedad, exhibiendo además, una variación continua en la población general (20).

Los endofenotipos contribuyen a discriminar en las enfermedades complejas, como lo son los trastornos mentales o la psicopatología, vías moleculares que subyacen a efectos genéticos que nos permiten reconocer patrones de susceptibilidad específicos (21). Con respecto a la literatura previa sobre familiares de primer grado de pacientes diagnosticados con $\mathrm{TB}$, los estudios realizados con poblaciones adultas han mostrado un déficit cognitivo global similar al observado en pacientes con TB, pero menos grave (22). En otros estudios han observado discrepancias significativamente mayores entre los índices de compresión verbal y el rendimiento en el grupo de niños cuyos padres presentaban la patología (23).

En la población colombiana se encontró un estudio que analiza el coeficiente intelectual, encontrando factores de riesgo que explicarían puntuaciones más bajas (24). Es importante resaltar de igual manera que la carga familiar por enfermedad psiquiátrica y adversidad en la infancia se conjugan para tener una influencia muy grande en la edad de inicio del trastorno bipolar, variables que deben considerarse en la evaluación del riesgo de aparición de enfermedades en diferentes poblaciones (25). De igual manera, se han reportado diferencias significativas entre el grupo de hermanos y el grupo de control en el factor de resolución de problemas y en la memoria de trabajo (26).

En estudios de la descendencia de esquizofrénicos se presenta peor rendimiento en la memoria de trabajo, mientras que la descendencia de bipolares se reporta puntuaciones significativamente más bajas en atención, ambas en comparación con grupo control (27). En otro estudio de descendientes de madres con TB, se reportan puntajes más bajos que en el grupo control en cuanto a coeficiente intelectual total, funciones ejecutivas, memoria espacial y atención. (28).

De los estudios más recientes realizados en hijos de padres con $\mathrm{TB}$, se encontró un rendimiento significativamente peor en la velocidad de procesamiento y la recuperación inmediata de la memoria visual. Cuando se analizó la presencia de cualquier psicopatología de por vida, los resultados mostraron que pertenecer al grupo de hijos de padres con TB fue el principal factor explicativo de las puntuaciones obtenidas tanto en la velocidad de procesamiento como en el recuerdo inmediato de la memoria visual, independientemente de la presencia de psicopatología (29).

En Colombia, un estudio describe los síntomas subumbrales más frecuentes en el grupo de los trastornos disruptivos y se observaron alteraciones en los dominios cognitivos como la atención, fluidez verbal, memoria de trabajo y velocidad de procesamiento de la información (30).

El presente estudio tiene como objetivo describir las características neuropsicológicas globales de niños y adolescentes descendientes de padres con antecedentes de Trastorno Bipolar respecto a la aplicación de una prueba estandarizada de inteligencia. Se determinó no 
incluir grupo control dado las características de la prueba neuropsicológica y su validación respectiva en español, para la población española y mexicana; además, en estudios colombianos se presenta como un instrumento confiable en la medición de la inteligencia y capacidad intelectual de niños y adolescentes escolares $(31,32$, 33). Igualmente, se pretende evaluar la influencia en el rendimiento escolar, independientemente de la presencia de psicopatología.

\section{Materiales y métodos:}

\section{Metodología:}

Estudio de tipo descriptivo, observacional, de corte transversal. Se evaluaron los sujetos cuyos padres padezcan de Trastorno Bipolar I; los sujetos evaluados mediante entrevista diagnóstica para estudios genéticos (DIGS) y, además, asisten al programa de Clínica Bipolar. Muestra: Sujetos entre 6 y 16 años de edad, hijos de pacientes con diagnóstico de Trastorno Bipolar I, muestreo por intención. Se aplicó una evaluación por medio de entrevista semiestructurada para recolectar los datos demográficos y clínicos. También se aplicaron varias subpruebas de la Escala de inteligencia de Weschler para niños, (WISC -IV) para determinar los índices de funcionamiento cognitivo en cuanto a (CIT, ICV, IRP, IMT, IVP).

\section{Criterios de inclusión y de exclusión:}

Inclusión al grupo de casos: Sujetos entre 6 y 16 años 11 meses al momento de realizar la entrevista y aplicación del test neuropsicológico. Se incluyen ambos sexos, de los hijos biológicos de padres con diagnóstico de Trastorno Bipolar I; (al menos uno de los dos).

Exclusión al grupo de casos: Menores con discapacidad visual/auditiva o historia de lesiones neurológicas estructurales y epilepsia. Menores que cumplan con criterios de discapacidad intelectual, trastornos del espectro autista, consumo de sustancias psicoactivas o se encuentren con psicopatología activa según criterios del DSM V.

\section{Instrumentos y recolección de datos:}

Se realizó historia clínica completa con énfasis en antecedentes psiquiátricos a través de entrevista estructurada (DIGS), aplicada previamente a los padres y entrevista semiestructurada (K-SADS LP), a los hijos participantes. Se aplicó la prueba Wechsler Intelligence Scale for Children, Fourth Edition. Administration and Scoring manual (WISC-IV) $(31,42)$ ya que es una de las pruebas de elección para valorar la inteligencia en niños y se encuentra validada en español la versión IV, (WISCIV) $(32,33,34)$. Esta prueba es para aplicar en niños y adolescentes con edades comprendidas entre 6 años 0 meses y 16 años 11 meses. El tiempo estimado para la aplicación del test es de 60-75 minutos (35).

\section{Procesamiento de los datos y análisis estadístico}

Para el procesamiento de los datos se utilizó el software SPSS versión 18, licenciado. El análisis univariado se realizó midiendo frecuencias para las variables cualitativas y medias de tendencia central y dispersión para las cualitativas. Posteriormente, se realizó análisis bivariado e inferencia mediante chi-cuadrado de Pearson (las variables son categóricas y dicotómicas, razón por la cual se utiliza ese estadígrafo) se utilizó un valor alfa de 0.05 . Posteriormente, se realizaron pruebas de normalidad para las variables numérica; T-student en los casos que cumplieron el criterio de normalidad. Para las que no tienen criterio de normalidad se aplicó el estadígrafo U-Mann-Whitney para muestras independientes (se tomó el valor de Shapiro- Wilk de las pruebas de normalidad).

\section{Componente ético}

Este trabajo cumple con las normas de investigación en seres humanos según lo dispuesto en la resolución No 008430 de 1993 del Ministerio de Salud y en la Declaración de Helsinki del año 2000 (36, 37). Se realizó, además, un asentimiento informado que deben diligenciar todos los menores de edad. El consentimiento informado fue aprobado por los comités de bioética de la Universidad de Caldas.

\section{Resultados}

En este estudio fueron seleccionados 40 individuos, de los cuales, al momento de la evaluación, uno presentaba diagnóstico de discapacidad intelectual, dos no desearon participar del estudio, tres no cumplieron con la edad a la hora de la aplicación de la prueba y cuatro presentaban consumo de sustancias psicoactivas. Cumplieron con los criterios de inclusión 30 de los hijos de personas con diagnóstico de TB I entre 6 y 16 años, con un promedio de edad de 11,2 años; el 50\% de ellos mujeres y $50 \%$ hombres. La mayoría de ellos $56,7 \%$ procedentes de Manizales, seguidos de Riosucio 10\%, Villamaría, Pácora, Chinchiná con un $6,7 \%$ cada uno respectivamente, y por último Aránzazu, Filadelfia, Salamina y Manzanares, cada uno con una participación del 3,3\%.

En el 53,3\% de los participantes no se reporta psicopatología, en el $26,7 \%$ se reportan trastornos de conducta, en el $10 \%$ trastorno por déficit de atención e hiperactividad (TDAH), en el 6,7\% depresión y en el restante 3,3\% ansiedad; los hallazgos clínicos fueron reportados durante la aplicación de la entrevista semiestructurada como antecedentes o síntomas subsindrómicos. 
En cuanto a los padres con diagnóstico de TB I, se identifica que en el 90\% de los participantes tenían a su madre con este diagnóstico, y el 10\% solo el padre, ninguno de ellos con ambos padres afectados. El promedio de edad de los progenitores con TB I corresponde a 36,5 años, con un mínimo de edad de 27 y un máximo de 51 años. El estado civil de la mayoría de los padres de los participantes, correspondía a separados $83,3 \%$, en unión libre 13,3\% y casados 3,3\%. El nivel de escolaridad de los padres con TB I corresponde a universidad 6,7\%, secundaria completa 63,3\%, secundaria incompleta $10 \%$ y primaria incompleta $20 \%$, los datos se presenta en la Tabla :

Tabla 1. Descriptores generales.

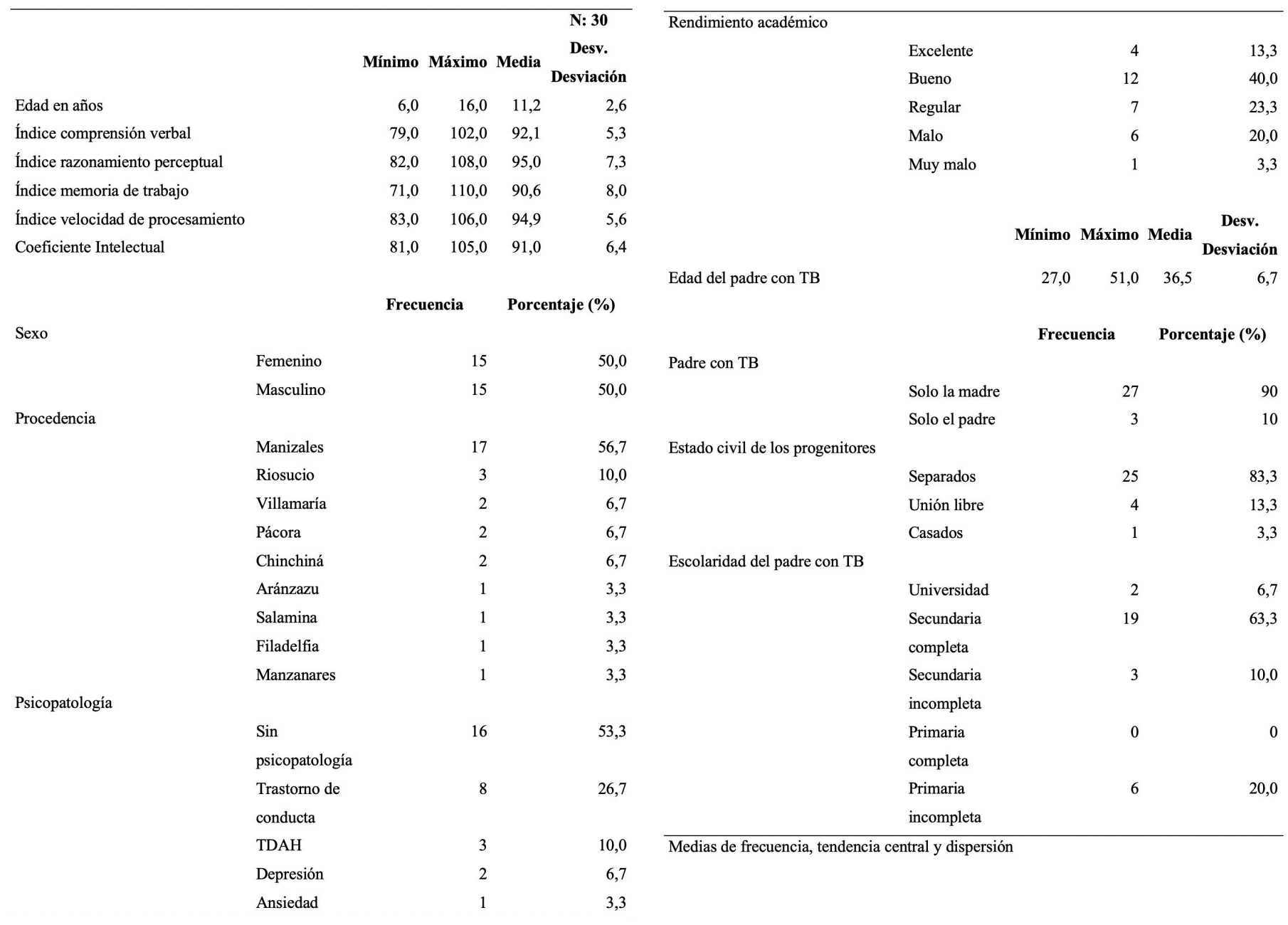

Al aplicar la prueba de inteligencia de Weschler para niños (WISC -IV), se obtuvieron los siguientes índices para la valoración de funciones cognitivas: a) Índice de comprensión verbal: se obtuvo un promedio de 92,1 con un mínimo de 79 y un máximo de 102, b) Índice de razonamiento perceptual: se obtuvo un promedio de 95 con un mínimo de 82 y un máximo de 108; c) Índice de memoria de trabajo: se obtuvo un promedio de 90,6 con un mínimo de 71 y un máximo de 110; d) Índice de velocidad de procesamiento: se obtuvo un promedio de 94,9 con un mínimo de 83 y un máximo de 106; e) Coeficiente Intelectual Total: se obtuvo un promedio de 91 con un mínimo de 81 y un máximo de 105.

Al analizar la tendencia central en los índices reportados para las funciones cognitivas, se identifica que la mejor respuesta corresponde al razonamiento perceptual, seguido de la velocidad del procesamiento, comprensión verbal y por último memoria de trabajo; así mismo, se indica que el índice con puntuación más baja en la valoración de las funciones cognitivas se encuentra también en la memoria de trabajo. Cabe señalar que el 36,6\% de los participantes en el estudio tienen un índice de comprensión verbal por debajo del promedio, igualmente; el 30\% un índice de razonamiento perceptual por debajo del promedio, el 43,3\% un índice de memoria de trabajo por debajo del promedio y un $16,6 \%$ también obtuvo un índice de velocidad de procesamiento por debajo del promedio, en relación a la curva de distribución normal establecido por la prueba aplicada. 
En cuanto al Coeficiente Intelectual Total, el 63,3\% de los participantes está por debajo del promedio de la curva de distribución normal, dada la estandarización del test, siendo 100 el promedio normal. Según la tendencia de la muestra de este estudio con un promedio de 91, un mínimo de 81 y máximo de 105; se clasifica la tendencia como un promedio normal bajo. Según la interpretación de la prueba de WISC - IV solo dos funciones (comprensión verbal y memoria de trabajo) obtuvieron índices por debajo de 80 pero superiores a 70, lo que según la prueba de Wechsler cualitativamente se asume como "límite". En la valoración de cada prueba, no se encontraron discrepancias entre los índices evaluados a nivel de cada participante. Respecto al rendimiento académico de los participantes en el estudio, debe advertirse que el $13,3 \%$ tienen un rendimiento excelente, el $40 \%$ bueno, el $23,3 \%$ regular, el $20 \%$ malo y el 3,3\% muy malo; en otros términos, lo anterior indica que el 53,3\% de los participantes tienen un rendimiento académico excelente o bueno; el restante $46,6 \%$ se caracteriza por un rendimiento académico regular, malo o muy malo.

En el análisis bivariado e inferencial de los índices por descripción cualitativa y datos cruzados según la prueba estandarizada de inteligencia aplicada se muestran en la Tabla 2:

Tabla. 2. Bivariados e inferencial.

Índices por descripción cualitativa según la prueba.

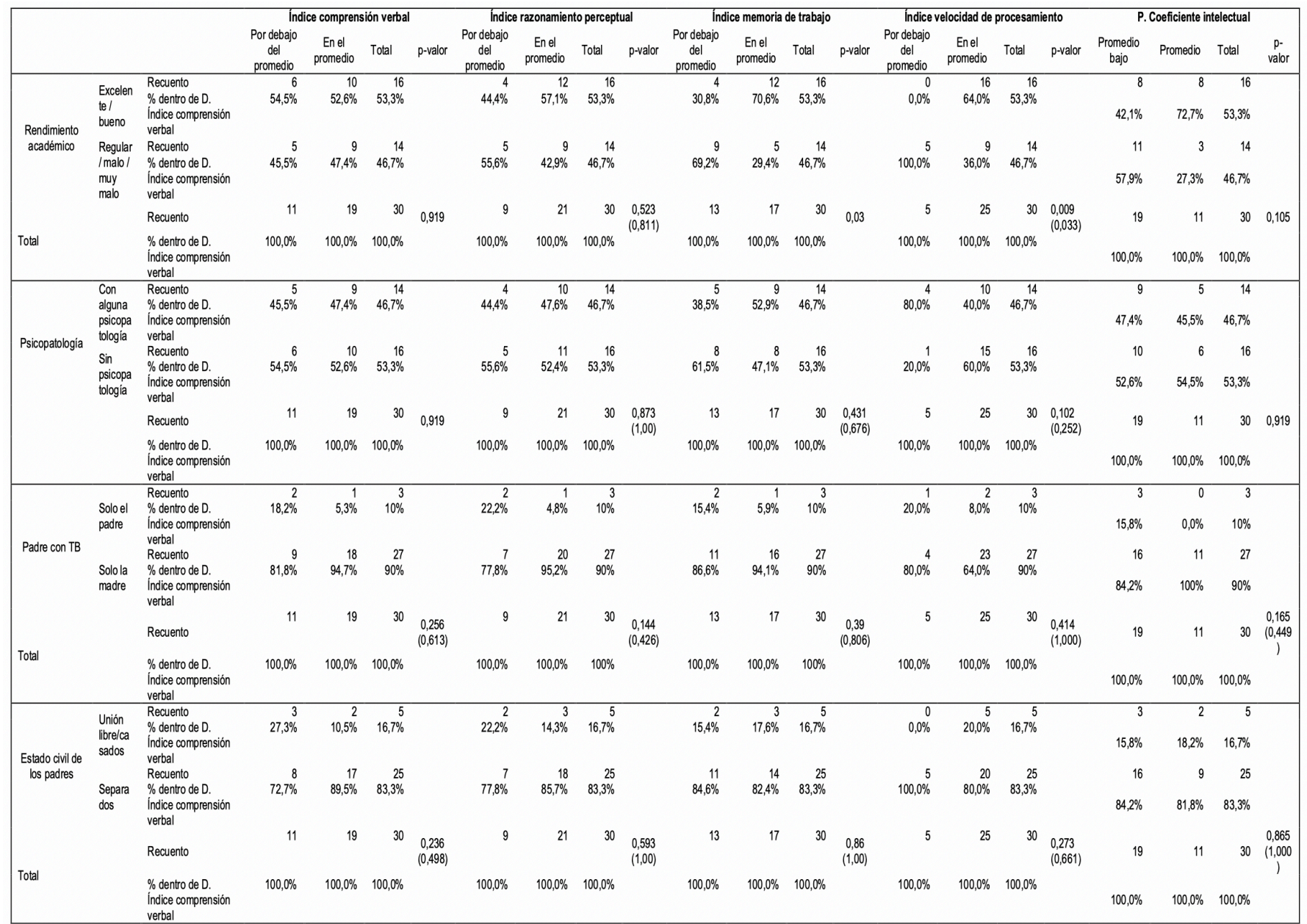

\section{a. Correlación de datos de participantes que obtuvieron un índice de comprensión verbal por debajo del promedio:}

1.Rendimiento escolar: el 54,5\% tiene un rendimiento académico excelente o bueno y el 45,5\% regular, malo o muy malo. De los que obtuvieron un índice promedio en esta prueba, el 56,2\% tienen un rendimiento académico excelente o bueno y el $47,4 \%$ un rendimiento regular, malo o muy malo. En consencuencia, no hay una relación estadísticamente significativa entre tener un índice de comprensión verbal por debajo del promedio y el mal rendimiento académico (regular, malo o muy malo) $(\mathrm{p}=0,919)$. 
2. Antecedente de psicopatología: el 45,5\% reportaron un antecedente de psicopatología y el 54,5\% no refirieron psicopatología. Por tanto, no hay una relación estadísticamente significativa entre tener un índice de comprensión verbal por debajo del promedio y el antecedente de psicopatología $(\mathrm{p}=0,919)$.

3. Progenitor con TBI: en el $81,8 \%$ solo la madre tenía como diagnóstico TB I y en el 18,2 \% solo el padre estaba diagnosticado con TB I. No se encontró una relación estadísticamente significativa entre tener un índice de comprensión verbal por debajo del promedio y el género del progenitor diagnosticado con TB I $(p=0,256)$.

4. Grupo familiar: en el $72,7 \%$ los padres estaban separados y en el $27,3 \%$ los padres estaban conviviendo juntos; sin embargo, no se encontró una relación estadísticamente significativa entre tener un índice de comprensión verbal por debajo del promedio y el estado civil de los padres $(\mathrm{p}=0,236)$.

b. Correlación de datos de población que obtuvo un índice de razonamiento perceptual por debajo del promedio:

1.Rendimiento académico: el $44,4 \%$ tiene un rendimiento académico excelente o bueno y el 55,6\% regular, malo o muy malo, por tanto, no hay una relación estadísticamente significativa entre tener un índice de razonamiento perceptual por debajo del promedio y el mal rendimiento académico (regular, malo o muy malo) $(\mathrm{p}=0,523)$.

2. Antecedente de psicopatología: el 44,4 \% reportaron un antecedente de psicopatología y el 55,6\% no refirieron psicopatología por lo que se establece que no hay una relación estadísticamente significativa entre tener un índice de razonamiento perceptual por debajo del promedio y el antecedente de psicopatología $(\mathrm{p}=$ $0,873)$.

3. Progenitor con TB I: en el 77,8 \% solo la madre tenía como diagnóstico TB I y en el $22,2 \%$ solo el padre estaba diagnosticado con TB I. No se encontró una relación estadísticamente significativa entre tener un índice de razonamiento perceptual por debajo del promedio y el género del progenitor diagnosticado con TB I $(p=1,44)$.

4. Grupo familiar: el 77,8 \% los padres estan separados y en el $22,2 \%$ los padres conviven juntos; sin embargo, no se encontró una relación estadísticamente significativa entre tener un índice de razonamiento perceptual por debajo del promedio y el estado civil de los padres $(\mathrm{p}=$ 0,593 ) c. Correlación de datos de población que obtuvo un índice de memoria de trabajo por debajo del promedio:

1. Rendimiento escolar: el 30,8\% tiene un rendimiento académico excelente o bueno y el 69,2 \% regular, malo o muy malo. Hay una relación estadísticamente significativa entre tener un índice de memoria de trabajo por debajo del promedio y el mal rendimiento académico (regular, malo o muy malo) $(\mathrm{p}=0,03)$.

2. Antecedente de psicopatología: el 38,5\% reportaron un antecedente de psicopatología y el $61,5 \%$ no refirieron psicopatología. No hay una relación estadísticamente significativa entre tener un índice de memoria de trabajo por debajo del promedio y el antecedente de psicopatología $(\mathrm{p}=0,431)$.

3. Progenitor con TB I: en el $84,6 \%$ solo la madre tenía como diagnóstico TB I y en el 15,4\% solo el padre estaba diagnosticado con TB I. No se encontró una relación estadísticamente significativa entre tener un índice de memoria de trabajo por debajo del promedio y el género del progenitor diagnosticado con TB I $(\mathrm{p}=0,390)$.

4. Grupo familiar: en el $84,6 \%$ los padres estaban separados y en el $15,4 \%$ los padres estaban conviviendo juntos; sin embargo, no se encontró una relación estadísticamente significativa entre tener un índice de memoria de trabajo por debajo del promedio y el estado civil de los padres $(\mathrm{p}=0,86)$.

d.Correlación de datos de población que obtuvo índice de velocidad de procesamiento por debajo del promedio:

1.Rendimiento escolar: todos tienen un rendimiento académico regular, malo o muy malo. Hay una relación estadísticamente significativa entre tener un índice de velocidad de procesamiento por debajo del promedio y el mal rendimiento académico (regular, malo o muy malo) $(\mathrm{p}=0,009)$.

2. Antecedente de psicopatología: el $80 \%$ reportaron un antecedente de psicopatología y el $20 \%$ no refirieron psicopatología. No hay una relación estadísticamente significativa entre tener un índice de velocidad de procesamiento por debajo del promedio y el antecedente de psicopatología $(\mathrm{p}=0,102)$.

3. Progenitor con TB I: en el $80 \%$ solo la madre tenía como diagnóstico TB I y en el $20 \%$ solo el padre estaba diagnosticado con TB I. No se encontró una relación estadísticamente significativa entre tener un índice de velocidad de procesamiento por debajo del promedio y el género del progenitor diagnosticado con TB I $(p=0,414)$. 
4. Grupo familiar: todos los participantes que obtuvieron un índice de velocidad de procesamiento por debajo del promedio, vivían con padres separados; sin embargo, no se encontró una relación estadísticamente significativa entre tener un índice de velocidad de procesamiento por debajo del promedio y el estado civil de los padres $(\mathrm{p}=0,273)$.

e. Correlación de datos de población que obtuvo un coeficiente intelectual por debajo del promedio en las puntuaciones de las subpruebas:

1.Rendimiento escolar: el 42,1\% tiene un rendimiento académico excelente o bueno y el 57,9\% regular, malo o muy malo. No hay una relación estadísticamente significativa entre tener un coeficiente intelectual por debajo del promedio y el mal rendimiento académico (regular, malo o muy malo) $(\mathrm{p}=0,105)$.

2. Antecedente de psicopatología: el 47,4\% reportaron un antecedente de psicopatología y el 52,6\% no refirió psicopatología. No hay una relación estadísticamente significativa entre tener un coeficiente intelectual por debajo del promedio y el antecedente de psicopatología $(\mathrm{p}=0,919)$.

3.Progenitor con TB I: en el 84,2 \% solo la madre tenía como diagnóstico TB I y en el 15,8\% solo el padre estaba diagnosticado con TB I. No se encontró una relación estadísticamente significativa entre tener un coeficiente intelectual por debajo del promedio y el género del progenitor diagnosticado con TB I $(\mathrm{p}=0,165)$.

4. Grupo familiar: en el $84,2 \%$ los padres estaban separados y en el $15,8 \%$ los padres estaban conviviendo juntos; sin embargo, no se encontró una relación estadísticamente significativa entre tener un coeficiente intelectual por debajo del promedio y el estado civil de los padres $(\mathrm{p}=0,865)$.

En cuanto a la población que obtuvo un índice de coeficiente intelectual con promedio normal bajo, en la mayoría $(63,2 \%)$ sus padres tienen un nivel de escolaridad de secundaria completa, seguido de un $21,1 \%$ cuyos padres tienen primaria incompleta, un $10,5 \%$ sus padres tienen como nivel secundario incompleta y por último un 5,3\% en el que sus padres tienen un nivel de escolaridad que corresponde a universidad. Es de aclarar que el nivel de escolaridad del progenitor con TB I no se relaciona con ninguno de los índices de las funciones cognitivas y tampoco con el coeficiente intelectual total (IC); ninguna prueba tuvo significancia estadística.

A continuación se presentan las pruebas de normalidad para las variables numéricas en la Tabla 3:

Tabla. 3. Pruebas de normalidad

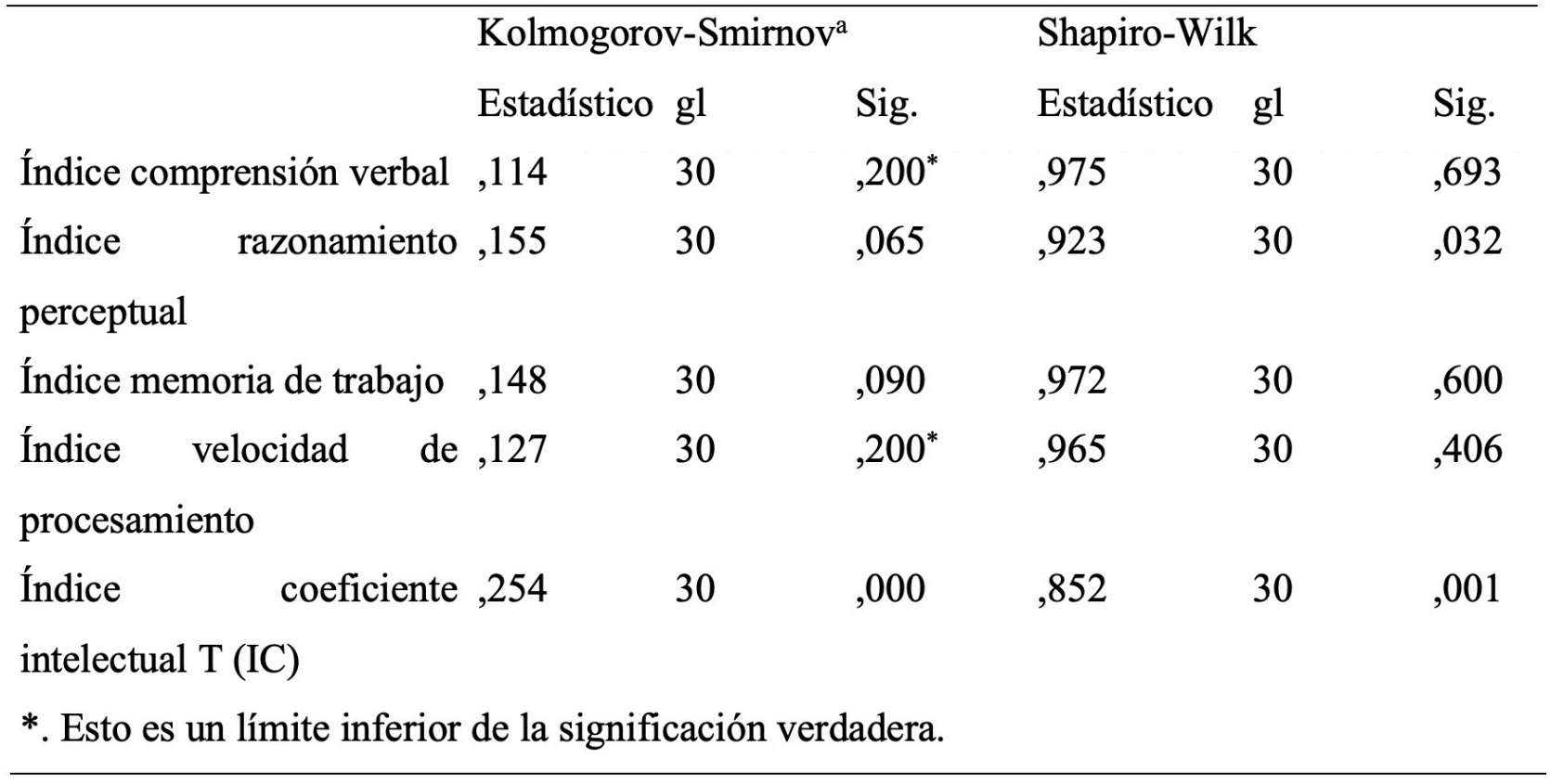




\section{a. Corrección de significación de Lilliefors}

Si la muestra es mayor a 30 se aplica Kolmogrow- Smirnov, si es de 30 o menos se aplica Shapiro- Wilk. Si p es mayor a 0,05 indica que existe distribución normal de los datos. * Para las que tienen distribución normal se aplica el estadígrafo t-student para muestras independientes (tomo el valor de Shapiro- Wilk).

En la Tabla 4 se presenta una comparación de medias (T-student) respecto a las pruebas que cumplen el criterio de normalidad: índices de compresión verbal, memoria de trabajo y velocidad de procesamiento:

Tabla. 4. Prueba T para la igualdad de medias con criterio de normalidad

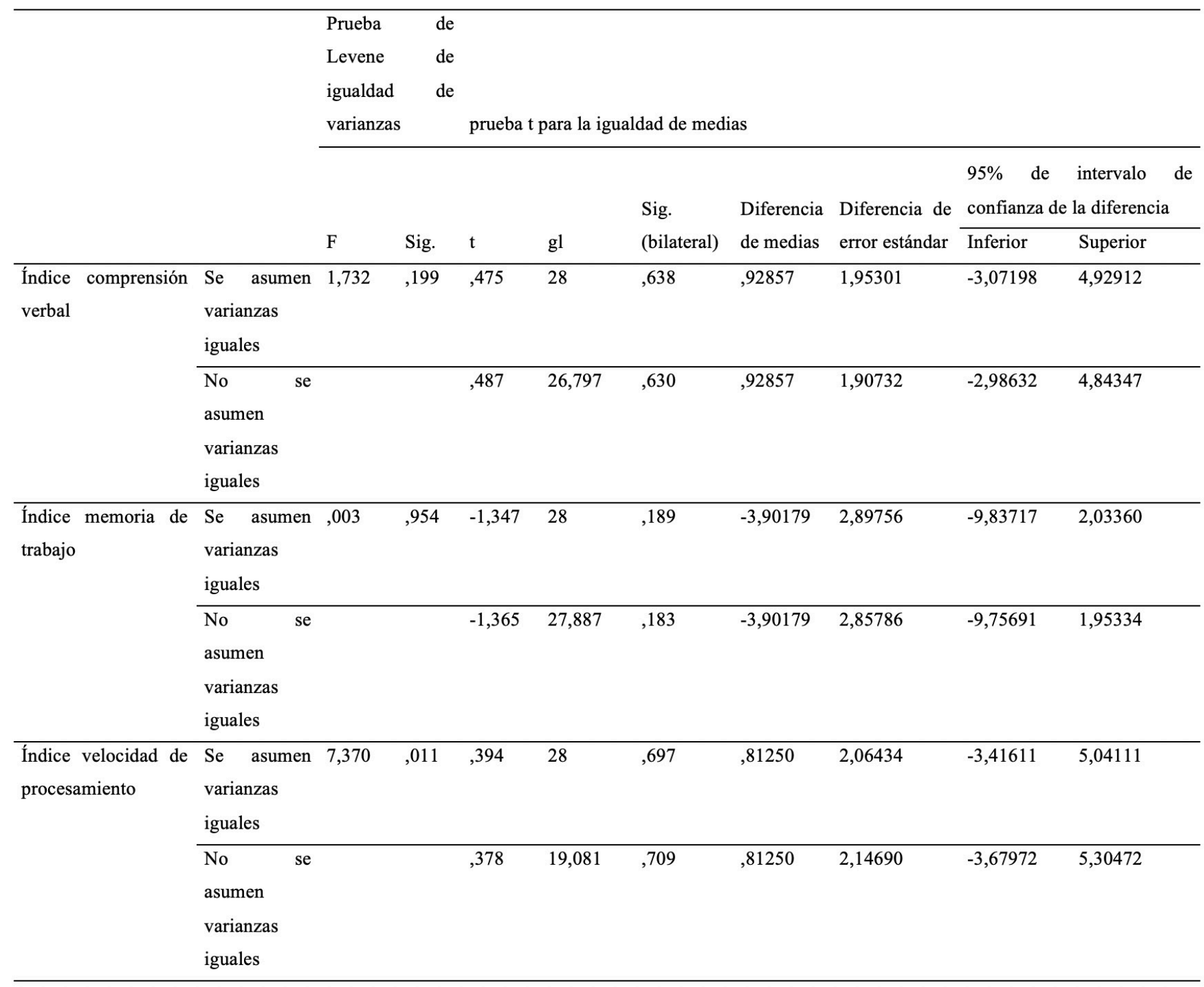

Para las pruebas que no tienen criterio de normalidad se aplicó el estadígrafo U-Mann-Whitney para muestras independientes (se tomó el valor de Shapiro- Wilk de las pruebas de normalidad), las variables fueron: índice de razonamiento perceptual e índice de coeficiente intelectual total y se tomó como variable dependiente la presencia o ausencia de psicopatología. Los datos se presentan en la Tabla 5: 
Tabla. 5. Prueba para la igualdad de medias sin criterio de normalidad

\begin{tabular}{lll}
\hline & Índice & razonamiento \\
& perceptual & Índice (ICT) \\
U de Mann-Whitney & 111,500 & 111,500 \\
W de Wilcoxon & 216,500 & 216,500 \\
$\mathrm{Z}$ &,- 021 &,- 021 \\
Sig. asintótica(bilateral) &, 983 &, 983 \\
Significación exacta & {$\left[2 *\left(\right.\right.$ sig., $984^{\mathrm{b}}$} &, $984^{\mathrm{b}}$ \\
unilateral)] & & \\
\hline
\end{tabular}

a. Variable de agrupación: D. Psicopatología

b. No corregido para empates

Según los resultados, se asumen varianzas iguales en los índices de comprensión verbal y memoria trabajo, sin embargo, no es diferente el índice de comprensión verbal y de memoria de trabajo entre las personas con psicopatología y sin psicopatología. Por otro lado, se asume que existen varianzas distintas para el índice de velocidad de procesamiento y diferencias en el índice de velocidad de procesamiento entre las personas con psicopatología y sin psicopatología; no obstante, estadísticamente no es significativo $(\mathrm{p}=0,709)$.

En cuanto a las variables que no cumplen criterio de normalidad, se encontró que no es diferente el índice de razonamiento perceptual en las personas con psicopatología y sin psicopatología $(p=0,983)$ al igual que el índice coeficiente intelectual total $(\mathrm{p}=0,983)$. Al no encontrar significancia estadística mediante la comparación de medias con los estadígrafos correspondientes para cada subprueba, no se realizan análisis de regresión logística binaria con variable resultado presencia de psicopatología.

\section{Discusión}

El presente estudio encontró en cuanto al Coeficiente Intelectual Total que el 63,3\% de los participantes está por debajo del promedio de la curva de distribución normal, según la estandarización del test de inteligencia aplicado (31, 32), donde se considera un rango de 90 a 110 como promedios normales. Comparando la tendencia de la muestra de este estudio con un promedio de 91, un mínimo de 81 y máximo de 105; se clasifica la tendencia de la muestra como un promedio normal bajo. No obstante, en la mayoría de los participantes se encuentra que las funciones cognitivas evaluadas están en el rango de normalidad (normal bajo).

Consistente con datos encontrados en la literatura donde algunos estudios en hijos de pacientes con TB evidencian un funcionamiento cognitivo global más bajo que la población general $(22,38,39)$, se estima que pertenecer a esta población aumentaría el riesgo a largo plazo de expresiones sintomáticas en la esfera cognitiva, independientemente de la presencia de la psicopatología a lo largo de la vida (40). Una explicación alternativa a este hallazgo podría ser que esta característica fuese propia de la población de la cual se tomó la muestra, posiblemente asociada a otros factores externos psicosociales, más que una característica inherente al estado de alto riesgo de psicopatología de estos sujetos.

Por otro lado, se obtuvieron en algunos participantes índices específicos por debajo de 80 pero superiores a 70 en comprensión verbal y memoria de trabajo, lo que según la prueba, cualitativamente, se asumiría como valor "límite", sin embargo, la evaluación global se encuentra en el rango normal bajo del funcionamiento cognitivo, este hallazgo nos indica que es posible encontrar funciones cognitivas específicas que impactan en el coeficiente intelectual de manera negativa, como la memoria de trabajo y en menor medida la compresión verbal, descrita ampliamente en otros estudios (17). Lo anterior, plantea la posibilidad de que las alteraciones en estos dominios pueden corresponder 
a un endofenotipo específico que se expresa de manera temprana en la población que sufre el trastorno y en sus descendientes $(20,21)$.

Por otro lado, al relacionar la memoria de trabajo $\mathrm{y}$ el rendimiento escolar se encuentra una relación estadísticamente significativa entre tener un índice de memoria de trabajo por debajo del promedio y el mal rendimiento académico $(\mathrm{p}=0,03)$, si bien no hay una relación estadísticamente significativa con la presencia o antecedentes de psicopatología, ni con el coeficiente intelectual total (23). Sin embargo, este resultado continúa siendo un inferencial, donde no es posible establecer una causalidad directa, ni controlar otras variables de carácter psicosocial que pueden conjugarse y sesgar la confiabilidad de los datos (24).

Se encontró, además, que hay una relación estadísticamente significativa entre tener un índice de velocidad de procesamiento por debajo del promedio y el mal rendimiento académico (regular, malo o muy malo) $(\mathrm{p}=0,009)$, sin embargo, en cuanto al impacto en la función cognitiva global fue mucho menor. En este sentido, hay que tener en cuenta que no se puede establecer una comparación directa o de causalidad.

De los participantes que obtuvieron un coeficiente intelectual por debajo del promedio, en el 84,2 \% la madre tenía el diagnóstico, sin embargo, no se encontró una relación estadísticamente significativa entre tener un coeficiente intelectual por debajo del promedio y el género del progenitor diagnosticado $(\mathrm{p}=0,165)$. Esto podría ser explicado por el diseño y recolección de datos de la muestra donde el $90 \%$ de los padres pertenecían al género femenino y se comportaban como cuidadores principales en la mayoría de los casos.

Adicionalmente, no se encontraron estudios que mostraran significancia estadística para el género del progenitor, siendo la evaluación del riesgo la presencia del trastorno en uno o ambos padres, con estimaciones entre el $15-30 \%$ para un padre afectado mientras que en el caso de que ambos padres padezcan TB el riesgo aumenta a $50-75 \%$ (8). En cuanto al número de padres afectados, no se obtuvieron datos con ambos progenitores con el diagnóstico.

En cuanto a la conformación familiar, los participantes que obtuvieron un coeficiente intelectual por debajo del promedio, en el $84,2 \%$ de los casos los padres estaban separados; sin embargo, no se encontró una relación estadísticamente significativa entre tener un coeficiente intelectual por debajo del promedio y el estado civil de los padres $(p=0,865)$. Resultado que llama la atención dado que, por el contrario de lo reportado en la literatura, la carga familiar por enfermedad psiquiátrica, adversidad en la infancia y carga genética se combinan para tener una influencia importante en la edad de inicio y presentación del trastorno bipolar (25).

Del total de personas con un índice de coeficiente intelectual con promedio bajo, la mayoría $(63,2 \%)$ de sus padres tienen un nivel de escolaridad de secundaria completa, seguido de un $21,1 \%$ cuyos padres tienen primaria incompleta, un $10,5 \%$ sus padres tienen como nivel secundario incompleta y por último un $5,3 \%$ en el que sus padres tienen un nivel de escolaridad que corresponde a universidad. Encontrando en una tercera parte de la muestra en los padres una escolaridad incompleta. Es de aclarar que el nivel de escolaridad del progenitor no se relacionó con ninguno de los índices de las funciones cognitivas y tampoco con el coeficiente intelectual total (IC); ninguna prueba tuvo significancia estadística. Sin embargo, estudios en la literatura reportan hallazgos especialmente, entre la categoría académica alcanzada por la madre y la índice comprensión verbal (41), lo cual puede ser llamativo, dado la importancia de la carga de enfermedad en los padres, su rendimiento escolar y los factores psicosociales que podrían estar relacionados.

En cuanto a los hallazgos psicopatológicos es importante resaltar que al momento de la entrevista y la aplicación de la prueba neuropsicológica no se encontraron síntomas activos, estos hallazgos se tuvieron en cuenta como antecedentes y síntomas subsindrómicos identificados con la aplicación de la entrevista semiestructurada. Palacio et al., en un estudio del 2016 en hijos de paciente con TB, describen una elevada tasa de psicopatología, siendo los trastornos del espectro afectivo, ansioso y el consumo de sustancias los más frecuentes. Estos sujetos presentaron un coeficiente intelectual promedio dentro de la normalidad. En nuestro estudio se encontró que los trastornos de la conducta y el trastorno por déficit de atención e hiperactividad son las expresiones psicopatológicas más frecuentes, no obstante, no se evidenciaron relaciones estadísticamente significativas en cuanto las puntuaciones en las subpruebas y en el coeficiente intelectual total, evaluados en las pruebas de normalidad de variables. De igual manera, no se encontraron asociaciones en cuanto al rendimiento escolar, género de los participantes, género del progenitor, ni su estado civil.

Al revisar la literatura se ha encontrado que el TB I presenta alteraciones específicas en algunos dominios neurocognitivos y que dichos daños también se podrían presentar en hijos sin la patología propiamente establecida por los episodios afectivos. Este estudio sugiere que un riesgo familiar en hijos de pacientes con TB I, puede contribuir a un incremento cuantitativo de las alteraciones cognitivas, especialmente en el índice de Memoria de Trabajo e Índice Compresión Verbal; lo cual podría incidir significativamente sobre las subpruebas 
del test neuropsicológico y ubicar los índices, según la distribución normal de los datos de las pruebas estandarizadas en un rango de normal bajo.

Estos hallazgos pueden sugerir un posible endofenotipo del TB I y su descendencia en relación a la presentación temprana de las alteraciones cognitivas en este grupo de riesgo, independientemente de la presencia de psicopatología. Al evaluar los participantes se encontró que en su totalidad estaban escolarizados y aproximadamente la mitad de la muestra presenta mal rendimiento escolar. Al asociar el rendimiento escolar con las subpruebas del test, se hallaron puntuaciones más bajas en relación a los índices de Memoria de Trabajo y Velocidad de Procesamiento. Por lo cual, sería importante en investigaciones futuras evaluar la correlación con factores psicosociales y psicopatológicos que puedan explicar los resultados. Dichos hijos pueden ser un punto de partida para realizar estudios que determinen predicción y realizar así un abordaje temprano.

Finalmente, como limitaciones de este estudio, se considera que al ser transversal y descriptivo solo permite mostrar asociaciones a través del análisis inferencial, pero no relación de causalidad, además presenta un tamaño de muestra pequeño, que corre el riesgo de presentar un error tipo II por múltiples comparaciones.

\section{Agradecimientos:}

Se agradece a los pacientes de la Clínica de Trastorno Bipolar de la Universidad de Caldas, y sus familiares.

\section{Referencias}

1. K.R. Merikangas,R. Jin,J.-P. He,R.C. Kessler,S. Lee,N.A. Sampson Prevalence and correlates of bipolar spectrum disorder in the world mental health survey initiative Arch. Gen. Psychiatry. 2011, 68: 241.

2. Kessler,R.C.,McGonagle,K.A.,Zhao,S.,Nelson, C. B., Hughes, M., Eshleman, S., Kendler, K. S. Lifetime and 12-month prevalence of DSM-V. Arch. Gen. Psychiatry. 1994; 51(1):8-19. Doi: 10.1001/archpsyc. 1994.03950010008002 . PMID: 8279933.

3. McGuffin, P., Rijsdijk, F., Andrew, M., Sham, P., Katz, R., \& Cardno, A. The heritability of bipolar affective disorder and the genetic relationship to unipolar depression. Arch. Gen. Psychiatry. 2013; 60(5): 497-502.

4. Lichtenstein, P., Yip, B. H., Björk, C., Pawitan, Y., Cannon, T. D., Sullivan, P. F., Hultman, C. M.. Common genetic determinants of schizophrenia and bipolar disorder in Swedish families: a population-based study. The Lancet. 2009. 373(9659): 234-239.

5. Jones, S. H., Bentall, R. P. A review of potential cognitive and environmental risk markers in children of bipolar parents. Clin. Psycho.l Rev. 2008; 28(7): 1083-1095.

6. Chang, K., Steiner, H., Ketter, T. Studies of offspring of parents with bipolar disorder. Am. J. Med. Genet. C. 2008; 123 (1): 26-35

7. Wray, N. R., Gottesman, I. I. Using summary data from the danish national registers to estimate heritabilities for schizophrenia, bipolar disorder, and major depressive disorder. Front. Genet. 2012; 3:118. DOI: 10.3389/fgene.2012.00118

8. Martínez, E. Trastorno bipolar, un mal silencioso. Periódico el Centroamericano. 25-09-2008.

9. Duffy, A., Grof, P., Robertson, C, Alda, M. The implications of genetics studies of major mood disorders for clinical practice. J. Clin. Psychiatry. 2000; 61(9):630-637.

10. DelBello, M. P., Geller, B. Review of studies of child and adolescent offspring of bipolar parents. Bipolar Disord. 2001; 3(6): 325-334.

11. Birmaher, B., Axelson, D., Monk, K., Kalas, C., Goldstein, B., Hickey, M. B., Kupfer, D. Lifetime psychiatric disorders in school-aged 
offspring of parents with bipolar disorder: the Pittsburgh Bipolar Offspring study. Arch. Gen. Psychiatry. 2009; 66(3):287-296.

12. Torres, I. J., Boudreau, V. G., Yatham, L. N. Neuropsychological functioning in euthymic bipolar disorder: a meta $\square$ analysis. Acta. Psychiatr. Scand. 2007;116: 17-26.

13. Lim, C. S., Baldessarini, R. J., Vieta, E., Yucel, M., Bora, E., Sim, K. Longitudinal neuroimaging and neuropsychological changes in bipolar disorder patients: review of the evidence. Neurosci. Biobehav. Rev. 2013;37(3):418-35. Doi: 10.1016/j.neubiorev.2013.01.003.

14. Clark, L., Iversen, S. D., Goodwin, G. M. Sustained attention deficit in bipolar disorder. Br. J. Psychiatry. 2002; 180(4): 313-319.

15. Martínez-Arán, A., Vieta, E., Reinares, M., Colom, F., Torrent, C., Sánchez-Moreno, J.,Salamero, M.Cognitive function across manic or hypomanic, depressed, and euthymic states in bipolar disorder. Am. J. Psychiatry. 2004; 161(2): 262-270.

16. Cahill, C. M., Walter, G., \& Malhi, G. S. Neurocognition in bipolar disorder and juvenile bipolar disorder. J. Can. Acad. Child. Adolesc. Psychiatry. 2009; 18(3):221.

17. Horn, K., Roessner, V., Holtmann, M. Neurocognitive performance in children and adolescents with bipolar disorder: a review. Eur. Child. Adolesc. Psychiatry. 2011; 20, 433-450.

18. Castañeda M, Tirado E, Feria M. Funcionamiento neuropsicológico de adolescentes con trastorno bipolar. Salud Mental. 2009; 32: 279-285.

19. Klimes-Dougan, B., Ronsaville, D., Wiggs, E. A. Martinez, P. Neuropsychological functioning in adolescent children of mothers with a history of bipolar or major depressive disorders. Biol. Psychiatry. 2006; 60(9): 957-965.

20. Gottesman, I., Gould, T. The endophenotype concept in psychiatry: etymology and strategic intentions. Am. J. Psychiatry.2003; 160(4): 636645.

21. Cuartas -Arias, J., Palacio- Acosta, C. Psychobiology and candidate endophenotypes in Psychopathy. Int.J.Psychol.Res. 2011. 4(1): 92-101.
22. Balanzá-Martínez, V., Rubio, C., Selva-Vera, G., Martinez-Aran, A., Sanchez-Moreno, J., SalazarFraile, J., Tabares-Seisdedos, R. Neurocognitive endophenotypes (endophenocognitypes) from studies of relatives of bipolar disorder subjects: a systematic review. Neurosci. Biobehav. Rev. 2008; 32(8): 1426-1438.

23. McDonough-Ryan, P., DelBello, M., Shear, P. K., Ris, M. D., Soutullo, C., Strakowski, S. M. Academic and cognitive abilities in children of parents with bipolar disorder: a test of the nonverbal learning disability model. J. Clin. Exp. Neuropsychol. 2002; 24(3): 280-285.

24. Zapata-Zabala, M. E., Álvarez-Uribe, M. C., Aguirre-Acevedo, D. C., Cadavid-Castro, M. A. Coeficiente intelectual y factores asociados en niños escolarizados en la ciudad de Medellín, Colombia. Revista de Salud Pública. 2012; 14: 543-557

25. Post, R. M., Altshuler, L. L., Kupka, R., McElroy, S. L., Frye, M. A., Rowe, M., Nolen, W. A. Age of onset of bipolar disorder: combined effect of childhood adversity and familial loading of psychiatric disorders. J. Psychiatr. Res. 2016; 81: 63-70.

26. Doyle, A. E., Wozniak, J., Wilens, T. E., Henin, A., Seidman, L. J., Petty, C., Biederman, J.. Neurocognitive impairment in unaffected siblings of youth with bipolar disorder. Psychol. Med. 2009; 39(8): 1253-1263.

27. Diwadkar, V. A., Goradia, D., Hosanagar, A., Mermon, D., Montrose, D. M., Birmaher, B., Zajac-Benitez, C. Working memory and attention deficits in adolescent offspring of schizophrenia or bipolar patients: comparing vulnerability markers. Prog Neuropsychopharmacol. Biol. Psychiatry. 2011; 35(5): 1349-1354.

28. de la Serna, E., Sugranyes, G., Sanchez-Gistau, V., Rodriguez-Toscano, E., Baeza, I., Vila, M., Castro-Fornieles, J. Neuropsychological characteristics of child and adolescent offspring of patients with schizophrenia or bipolar disorder. Schizophr. Res. Treatment. 2017; 183: 110-115.

29. Palacio-Ortíz, J. D., Uribe-Villa, E., DuqueRíos, P., Gutiérrez-Briceño, P., Zapata-Henao, V., Peña-Quintero, C. E., López-Jaramillo, C. Descripción de perfiles psicopatológicos y neurocognitivos de hijos de pacientes con trastorno bipolar tipo I dentro un programa de intervencion multimodal: PRISMA. Rev. 
Colombiana de Psiquiatría. 2015; 44(3):150158.

30. Corral S, Arribas D, Santamaría P, Sueiro M.J., Pereña J. Escala de inteligencia de Weschler para niños (WISC -IV). Consejo general de colégios oficiales de psicólogos. 2005.

31. Contreras, D. M. M., \& Rodríguez, Á. P. A.. Estudio preliminar de las propiedades psicométricas del WISC-IV en una muestra de escolares de Bucaramanga. Informes Psicológicos. 2013; 13(2):13-25.

32. Carreño Novoa, L. S. Calidad de la adaptación y traducción del WISC-IV para la población colombiana. En: IV Encuentro Latinoamericano de Metodología de las Ciencias Sociales 27 al 29 de agosto de 2014 Heredia, Costa Rica. Universidad Nacional de La Plata. Facultad de Humanidades y Ciencias de la Educación. Centro Interdisciplinario de Metodología de las Ciencias Sociales.

33. Corral S, Arribas D, Santamaría P, Sueiro M.J., Pereña J. Escala de inteligencia de Weschler para niños (WISC -IV). Consejo general de colegios oficiales de psicólogos. 2005.

34. Wechsler, D. Escala Wechsler de inteligencia para niños-IV. Manual moderno. 2005

35. Ministerio de Salud, Colombia. Dirección de Desarrollo Científico y Tecnológico. Normas científicas, técnicas y administrativas para la investigación en salud: resolución No. 008430 de 1993. 1993.

36. Manzini, J. L. Declaración de Helsinki: principios éticos para la investigación médica sobre sujetos humanos. Acta bioethica. 2000; 6(2): 321-334.

37. Fernández-Rodríguez,A. Perfil neuropsicológico del Trastorno Bipolar en niños y adolescentes. 2018.

38. Ribeiro-Fernández, M. Estabilidad diagnóstica y características del Trastorno Bipolar de inicio en la infancia y adolescencia en una muestra europea. 2019.

39. Toulopoulou, T., Quraishi, S., McDonald, C.,Murray, R. M. The Maudsley Family Study: premorbid and current general intellectual function levels in familial bipolar I disorder and schizophrenia. J. Clin. Exp. Neuropsychol. 2006; 28(2):243-259.
40. Labin, A., Brenlla, M. E.,Taborda, A. La relación entre el nivel educativo de la madre y el rendimiento cognitivo infanto-juvenil a partir del WISC-IV. Psicogente, 18(34).

41. Kaufman, J., Birmaher, B., Axelson, D., Perepletchikova, F., Brent, D., Ryan, N. K-SADS-PL DSM-5. Pittsburgh: Western Psychiatric Institute and Clinic. 2016 